\title{
ON ANGULAR MOMENTUM HELMHOLTZ THEOREMS AND COHOMOLOGY OF LIE ALGEBRAS
}

\author{
BY
}

\section{HENRIK STETKAER}

ABSTRACT. Helmholtz' 2nd theorem (that every vector field on $\mathrm{R}^{3}$ with vanishing curl is gradient of a function) can be viewed as a statement about the group of translations of $R^{3}$. We prove similar theorems for other Lie transformation groups, in particular for semidirect products of abelian and compact semisimple groups. Using Hodge theory we also obtain results analogous to the 1st Helmholtz theorem, but only for compact Lie transformation groups.

I. Introduction. The two Helmholtz theorems are standard tools of vector analysis. The first theorem states that every smooth vector field $\Phi$ on $\mathbf{R}^{\mathbf{3}}$ in exactly one way can be decomposed into two terms

$$
\Phi=\nabla F+\operatorname{curl} \vec{V}
$$

where $F \in C^{\infty}\left(\mathrm{R}^{3}\right)$ and where $\vec{V}$ is a smooth vector field on $\mathrm{R}^{3}$. The second states that a smooth vector field $\Phi$ on $\mathbf{R}^{3}$ is of the form $\Phi=d F$ provided curl $\Phi=0$.

In [14] Lomont and Moses obtained results that were quite analogous to the two Helmholtz theorems above, replacing the linear momentum operator $\nabla$ by the angular momentum operator $\vec{L}=\vec{x} \times \nabla$. A simpler and more direct proof was presented by J. B. Keller [10]. Their results were extended further by Cannon and Jordan [3] who studied, instead of vector fields, i.e. maps of $\mathbf{R}^{\mathbf{3}}$ into $\mathbf{R}^{\mathbf{3}}$, maps of $\mathbf{R}^{3 N}$ into $\mathbf{R}^{\mathbf{3}}$.

In connection with an application [13] of the above results to the no-interaction theorem of particle dynamics, $H$. Leutwyler observed that the second Helmholtz theorem and its analogs are "of a purely group-theoretical nature and have an immediate generalization to a large class of Lie groups" [12, p. 544]. Group theory enters because the operators $\nabla$ and $\vec{L}$ are the infinitesimal generators of the translation group in $\mathbf{R}^{3}$ and of the rotation group SO(3). Leutwyler's demonstration of his generalization-hereafter referred to as Leutwyler's theoremcan be found in [12].

Received by the editors December 6, 1974.

AMS (MOS) subject classifications (1970). Primary 18H25, 57E15, 57E20; Secondary 22E70, 53A45, 57F10.

Key words and phrases. Differential 1-forms, Helmholtz theorems, Hodge theory, Lie algebra cohomology, Lie transformation group. 
The purpose of the first part of this paper is to extend Leutwyler's theorem (as stated in §II) to more general actions of Lie groups and to give coordinate free and structure constant free proofs. The results can be stated as follows:

Let $G$ be a Lie transformation group of a manifold $M$. Let $g$ denote the Lie algebra of $G$.

In §III we show (Theorem 1) that Leutwyler's result is equivalent to $H^{1}\left(g, C^{\infty}(M)\right)=0$.

The main result of $\S I V$ is Theorem 2 which generalizes a theorem due to van Est [6]. It can be stated as follows:

Let $F$ be a weakly $C^{\infty} G$-module and assume the first de Rham cohomology group of $G$ vanishes. Then $H^{1}(G, F)$ and $H^{1}(g, F)$ are isomorphic vector spaces, not just when $F$ is finite-dimensional as in [6], but even when $F$ is a quasi-complete locally convex topological vector space. Leutwyler's original theorem is a consequence of Theorem 2 with $F=C^{\infty}(M)(\S \mathrm{V}$, Corollary 5).

$\S$ VI presents a simple way of handling Leutwyler's problem in the case where $G$ acts on a product of homogeneous $G$-spaces.

The second part of the paper ( $\S \mathrm{VII}-\mathrm{IX})$, which is essentially independent of the first one, generalizes the angular momentum first Helmholtz theorem to any actions of compact Lie groups, not just the action of $S O(3)$ on $C^{\infty}\left(R^{3}\right)$ as in the Lomont-Moses case.

With the notation above, the problem can be phrased: How to decompose any linear map $\Phi: g \rightarrow F$ in a way consistent with the action of $G$ on $F$ ? This is, of course, a generalization of the problem in the first part of the paper where we impose the additional assumption that $\Phi$ should be a cocycle, since we there wanted to show that $H^{1}(g, F)=0$.

To see the connection with Helmholtz' first theorem, we note that any vector field $\Phi$ on $\mathrm{R}^{3}$ via its components $\Phi_{i} \in C^{\infty}\left(\mathrm{R}^{3}\right), i=1,2,3$, can be identified with a linear map $\Phi: \mathbf{R}^{3} \rightarrow C^{\infty}\left(\mathbf{R}^{3}\right)$, namely the one determined by

$$
\Phi\left(e_{i}\right)=\Phi_{i} \text { for } i=1,2,3,
$$

where $\left(e_{1}, e_{2}, e_{3}\right)$ is a basis of $\mathbf{R}^{3}$. Helmholtz' first theorem may thus be interpreted as a result about how one can decompose the linear maps from $\mathbf{R}^{3}$ ( $=$ the Lie algebra of the group of translations of $R^{3}$ ) into $C^{\infty}\left(R^{3}\right)$. Similarly the corresponding Lomont-Moses theorem can be interpreted as a result about how to decompose any linear map $\Phi: s o(3) \rightarrow C^{\infty}\left(\mathbf{R}^{3}\right)$ in a way consistent with the action of $S O(3)$.

Our contribution, which encompasses the above-mentioned results of [3] and [14] (see Example 9 of §IX), can be summarized as follows:

Let there be given a strongly continuous, weakly $C^{\infty}$ representation of a compact Lie group $G$ on a quasi-complete, locally convex topological vector space 
$F$. This induces a $g$-module structure on $F$, where $g$ is the Lie algebra of $G$.

Then every linear map $\Phi: g \rightarrow F$ can in exactly one way be decomposed into three terms,

$$
\Phi=d F+\delta \Psi+\Theta
$$

where $d$ is the coboundary operator on the g-module $F, F \in F, \delta$ is the codifferential operator corresponding to $d$ under a given inner product on $g, \Psi: g \times g \rightarrow F$ is an alternating bilinear map, and $\Theta: g \rightarrow F$ is a harmonic linear map.

If, in particular, $G$ is semisimple as in the Lomont-Moses case where $G=$ $S O(3)$, then the harmonic term vanishes.

The main idea of the proof is to use Hodge theory to decompose the differential 1 -forms on $G$ and then to transfer the decomposition to the F-valued 1 -forms on $g$.

The author would like to thank P. Kristensen from the Physics Department for drawing his attention to Leutwyler's results, and to thank Verner Beck and $\mathrm{J} \phi$ rgen Tornehave for illuminating discussions.

II. The notation and Leutwyler's problem. Throughout this paper $G$ denotes an $n$-dimensional Lie group with identity element $e$, Lie algebra $g$ and exponential map exp: $g \rightarrow G$. We assume furthermore that $G$ acts as a Lie transformation group on a manifold $M$ (for definition, see [7, Chapter II, §3]), where by a manifold we will always understand a paracompact $C^{\infty}$ manifold. The action of $g \in G$ on $p \in M$ is written $g p$.

If $X \in g$ and $g \in G$ we let $X_{g}=L(g)_{*} X$, where $L(g)$ denotes left translation in $G$ by $g \in G$.

Each $X \in g$ induces a vector field $X^{*}$ on $M$ by

$$
X_{m}^{*}(f):=\left.\frac{d}{d t} f(\exp (-t X) m)\right|_{t=0} \quad \text { for } m \in M, f \in C^{\infty}(M)
$$

and it is well known that the map $X \mapsto X^{*}$ is a Lie algebra homomorphism of $g$ into the Lie algebra of $C^{\infty}$ vector fields on $M$ (see for example [15, p. 34]).

To state Leutwyler's problem we let $\left\{X_{1}, \ldots, X_{n}\right\}$ be a basis for $g$ and introduce the corresponding structure constants $c_{A B}^{C}$ where $A, B, C=1,2, \ldots, n$ by

$$
\left[X_{A}, X_{B}\right]=\sum_{C=1}^{n} c_{A B}^{C} X_{C}
$$

We shall examine whether the system

$$
X_{A}^{*} F=F_{A}, \quad A=1, \ldots, n,
$$

of 1 st order partial differential equations has a solution $F \in C^{\infty}(M)$ given $F_{1}$, $\ldots, F_{n} \in C^{\infty}(M)$. It follows immediately from (1) that a necessary condition 
for a solution to exist consists of the compatibility conditions

$$
X_{A}^{*} F_{B}-X_{B}^{*} F_{A}=\sum_{C=1}^{n} c_{A B}^{C} F_{C} \quad \text { for } A, B=1, \ldots, n \text {. }
$$

LEUTWYLER'S PROBLEM. Has (2) solutions $F \in C^{\infty}(M)$ for any $F_{1}, \ldots, F_{n}$ $\in C^{\infty}(M)$ satisfying (3)?

We will say that Leutwyler's problem can be solved if the answer to this question is yes.

Simple examples show that the Leutwyler's problem in general cannot be solved, so the question we ask is: What conditions on $G$ and $M$ guarantee that Leutwyler's problem can be solved? Leutwyler, himself, considered [12] the following types of Lie groups:

(a) groups with compact covering group,

(b) translation groups,

(c) semidirect products of (b) with (a).

He assumed furthermore that $M$ was a vector space with $G$ acting as a group of linear transformations. His result may, in the terminology above, be phrased as follows:

LEUTWYLER'S THEOREM. If $G$ is of type (a), (b) or (c), and if the translations are represented injectively in the cases (b) and (c), then Leutwyler's problem can be solved.

III. Reformulation of Leutwyler's problem. We proceed to reformulate Leutwyler's problem in cohomological terms. To make the exposition more selfcontained we recall the definition of the 1 st (Hochschild) cohomology group of a Lie algebra $g$ with respect to a $g$-module $F$. For more information we refer the reader to [9]. The action of $X \in g$ on $F \in F$ will be denoted $X \cdot F$.

Definition 1. A linear map $\Phi: g \rightarrow F$ is said to be a cocycle if

$$
X_{1} \cdot \Phi\left(X_{2}\right)-X_{2} \cdot \Phi\left(X_{1}\right)-\Phi\left(\left[X_{1}, X_{2}\right]\right)=0 \text { for all } X_{1}, X_{2} \in g \text {. }
$$

It is said to be a coboundary if there is an $F \in F$ such that $\Phi=\delta F$, meaning

$$
\Phi(X)=X \cdot F \quad \text { for all } X \in g \text {. }
$$

It is easy to see that the vector space $B^{1}(g, F)$ of coboundaries is a subspace of the vector space $Z^{1}(g, F)$ of cocycles. We may thus define the 1st cohomology group $H^{1}(g, F)$ as the quotient vector space

$$
H^{1}(g, F):=Z^{1}(g, F) / B^{1}(g, F) .
$$

Finally we will use the notation

$$
H^{0}(g, F)=\{F \in F \mid X \cdot F=0 \text { for all } X \in g\} .
$$


We shall now use the cohomology above in the special case where $F=C^{\infty}(M)$ and the g-module action is

$$
X \cdot F=X^{*} F \quad \text { for } F \in C^{\infty}(M) .
$$

Consider for $F_{1}, \ldots, F_{n} \in C^{\infty}(M)$ the linear map $\Phi: g \rightarrow C^{\infty}(M)$ determined by

$$
\Phi\left(X_{A}\right)=F_{A} \quad \text { for } A=1, \ldots, n \text {. }
$$

Two small computations show that

$\Phi$ is a cocycle if and only if (3) holds, and

$\Phi$ is a coboundary if and only if there exists an $F \in C^{\infty}(M)$ such that (2) holds.

Hence Leutwyler's problem can be solved if and only if every cocycle is a coboundary, so we have proved

THEOREM 1. Leutwyler's problem can be solved if and only if

$$
H^{1}\left(g, C^{\infty}(M)\right)=\{0\} .
$$

Note that the basis $\left\{X_{1}, \ldots, X_{n}\right\}$ for $g$ does not occur in (4) so that Leutwyler's problem only depends on the action of $g$ on $C^{\infty}(M)$ and not on any choice of basis.

IV. A general theorem. The main result of this section is Theorem 2 which has Leutwyler's theorem as a corollary (see §V).

$F$ will from now on denote a locally convex topological vector space over the reals with topological dual $F^{\prime}$. It is a standard result that $F^{\prime}$ separates the points of $F$. The pairing between $F^{\prime}$ and $F$ will be written $\langle\cdot, \cdot\rangle$.

We assume given a strongly continuous representation of the Lie group $G$ on $F$ by continuous linear operators; the action of $g \in G$ on $F \in F$ will be written $g \cdot F$. Note that we get an action of $G$ on $F^{\prime}$ (the contragredient representation), defined by

$$
\langle g \cdot u, F\rangle:=\left\langle u, g^{-1} \cdot F\right\rangle \text { for } u \in F^{\prime}, F \in F, g \in G .
$$

We will finally assume that $G$ "acts weakly $C^{\infty}$ on $F$ " in the sense that the map $g \mapsto g \cdot F$ of $G$ into $F$ for each fixed $F \in F$ is weakly $C^{\infty}$, where by "weakly $C^{\infty}$ " we mean:

Definition 2. A map $\varphi: G \rightarrow F$ is said to be weakly $C^{\infty}$ if

(a) $\langle u, \varphi\rangle \in C^{\infty}(G)$ for each $u \in F^{\prime}$.

(b) To each differential operator $D$ on $G$ with $C^{\infty}$ coefficients there exists a map $\varphi_{D}: G \rightarrow F$ such that

$$
D\langle u, \varphi\rangle=\left\langle u, \varphi_{D}\right\rangle \text { for all } u \in F^{\prime} .
$$


Definition 3. Let $\varphi: G \rightarrow F$ be weakly $C^{\infty}$. The differential $d \varphi$ of $\varphi$ at $e \in G$ is the linear map $d \varphi: g \rightarrow F$ which is determined by

$$
\langle u, d \varphi(X)\rangle=X\langle u, \varphi\rangle \quad \text { for all } X \in g \text { and } u \in F^{\prime} .
$$

Let us, for $F \in F$ and $X \in g$, define $X \cdot F \in F$ as the differential of the map $g \mapsto g \cdot F$.

Note the formula

$$
X_{g}(h \mapsto\langle u, h \cdot F\rangle)=\langle u, g \cdot X \cdot F\rangle \quad \text { for } g \in G, F \in F, u \in F^{\prime},
$$

which as a consequence has that the map $(X, F) \mapsto X \cdot F$ of $g \times F$ into $F$ makes F into a g-module.

REMARK. It is not necessary above or in the sequel that $u$ ranges over all of $F^{\prime}$; an inspection of the proofs below shows that a fixed invariant subset that separates the points of $F$ will do.

EXAMPLE 1. With the notations of $\S I I$ we let $F=C^{\infty}(M)$, where $C^{\infty}(M)$ always will be equipped with its standard topology (uniform convergence on compacta in $M$ of functions and their derivatives of all orders), making it into a Montel space. The Lie group $G$ acts on $C^{\infty}(M)$ by

$$
(g \cdot F)(m):=F\left(g^{-1} m\right) \text { for all } g \in G, m \in M \text {. }
$$

It is easy to check that all the assumptions above hold. In particular we find

$$
X \cdot F=X^{*} F \quad \text { for all } X \in g, F \in F .
$$

Definition $3^{\prime}$. An F-cocycle $\varphi$ is a weakly $C^{\infty}$ map $\varphi: G \rightarrow F$ satisfying

$$
\varphi(g h)=\varphi(g)+g \cdot \varphi(h) \text { for all } g, h \in G \text {. }
$$

An F-coboundary $\varphi$ is a map $\varphi: G \rightarrow F$ of the form

$$
\varphi(g)=g \cdot F-F \text {, where } F \text { is an element of } F \text {. }
$$

Each $F$-coboundary is an F-cocycle. The quotient vector space between the vector space of $F$-cocycles and the vector subspace of $F$-boundaries $B^{1}(G, F)$ will here be denoted $H^{1}(G, F)$.

LEMMA 1. If $\varphi: G \rightarrow F$ is an F-cocycle (-coboundary), then its differential $d \varphi: g \rightarrow F$ is a cocycle (coboundary).

Furthermore we have the formula

$$
X_{g}\langle u, \varphi\rangle=\langle u, g \cdot d \varphi(X)\rangle \text { for all } u \in F^{\prime}, X \in g, g \in G .
$$

ProOF. (8) is a simple consequence of (6). Using it we have 


$$
\begin{aligned}
\langle u, d \varphi([X, Y])\rangle & =[X, Y]\langle u, \varphi\rangle \\
& =X\left(g \mapsto Y_{g}\langle u, \varphi\rangle\right)-Y\left(g \mapsto X_{g}\langle u, \varphi)\right) \\
& =X(g \mapsto\langle u, g \cdot d \varphi(Y)\rangle)-Y(g \mapsto\langle u, g \cdot d \varphi(X)\rangle) \\
& =\langle u, X \cdot d \varphi(Y)\rangle-\langle u, Y \cdot d \varphi(X)\rangle,
\end{aligned}
$$

which shows that $d \varphi$ is a cocycle.

If $\varphi$ is a coboundary, i.e. of the form $\varphi(g)=g \cdot F-F$ for some $F \in F$, then it follows immediately by the very definition of $X \cdot F$ that $d \varphi(X)=X \cdot F$ for all $X \in g$.

Definition 4. According to Lemma 1 we may without ambiguity define a linear map $I: H^{1}(G, F) \rightarrow H^{1}(g, F)$ by

$$
I\left(\varphi+B^{1}(G, F)\right):=d \varphi+B^{1}(g, F) .
$$

Proposition 1. I is an injection. In particular, if $H^{1}(g, F)=0$, then $H^{1}(G, F)=0$.

Proof. If $d \varphi$ is a coboundary, i.e. $d \varphi(X)=X \cdot F$ for some $F \in F$ and all $X \in g$, then we find again by (8) that

$$
X_{g}\langle u, \varphi(\cdot)\rangle=\langle u, g \cdot(X \cdot F)\rangle .
$$

But $X_{g}(h \mapsto\langle u, h \cdot F-F\rangle)=\langle u, g \cdot(X \cdot F)\rangle$, so

$$
X_{g}\{\langle u, \varphi(\cdot)\rangle-\langle u,(\cdot) \cdot F-F\rangle\}=0 .
$$

Hence, $\langle u, \varphi(\cdot)\rangle-\langle u,(\cdot) \cdot F-F\rangle=$ constant. Since the left-hand side vanishes at $e \in G$, the constant is 0 , so we find $\varphi(g)=g \cdot F-F$ for all $g \in G$, showing $\varphi$ is a coboundary.

We will later (Theorem 2) show that $I$ under mild conditions is surjective as well, but first we want to digress a little and analyze the cocycle condition.

Let us recall the few basic facts about integration of vector valued functions that we will need shortly:

Let $T$ be a compact Hausdorff space with a Radon measure $\mu$, and let $f: T \rightarrow F$ be a continuous function. Then we define $\int_{T} f d \mu \in F^{*}$ by

$$
\left\langle u, \int_{T} f d \mu\right\rangle:=\int_{T}\langle u, f(s)\rangle d \mu(s) \text { for all } u \in F^{\prime} .
$$

If $F$ is quasi-complete, then $\int_{T} f d \mu$ actually belongs to $F$ by [1, Corollaire, p. 12] and [11,(3), p. 241]. Furthermore, if $f$ depends on a parameter $\omega$ in a topological space $\Omega$ in such a way that $f: T \times \Omega \rightarrow F$ is continuous, then $\omega \mapsto$ $\int_{T} f(s, \omega) d \mu(s)$ is a continuous map of $\Omega$ into $F$.

Let $L(F, F)$ denote the vector space of all continuous linear operators in $F$. Assume $\rho: T \rightarrow L(F, F)$ is such that the map $(t, F) \mapsto \rho(t) F$ of $T \times F$ into $F$ is 
continuous. If again $F$ is quasi-complete, we can define a continuous linear map $\int_{T} \rho(t) d \mu(t) \in L(F, F)$ by

$$
\int_{T} \rho(t) d \mu(t) F=\int_{T} \rho(t) F d \mu(t) \quad \text { for } F \in F .
$$

(Cf. [1, Lemma 3, p. 26 and its preceding remarks].)

Proposition 2. Suppose $F$ is quasi-complete. Then every F-cocycle is continuous. Conversely, any continuous map $\varphi: G \rightarrow F$ satisfying (6) is an F-cocycle.

Proof. First let $\varphi$ be an F-cocycle. By (6) it suffices to prove $\varphi$ is continuous at $e \in G$, i.e. that the map $X \mapsto \varphi(\exp (X))$ of $g$ into $F$ is continuous. Now (8) implies for any $u \in F^{\prime}$ that

$$
\begin{aligned}
\langle u, \varphi(\exp X)\rangle & =\int_{0}^{1} \frac{d}{d t}\langle u, \varphi(\exp (t X))\rangle d t=\int_{0}^{1} X_{\exp (t X)}\langle u, \varphi(\cdot)\rangle d t \\
& =\int_{0}^{1}\langle u, \exp (t X) \cdot d \varphi(X)\rangle d t=\left\langle u, \int_{0}^{1} \exp (t X) \cdot d \varphi(X) d t\right\rangle,
\end{aligned}
$$

where the last equality sign is justified because the map $t \mapsto \exp (t X) \cdot d \varphi(X)$ is continuous from $[0,1]$ to $F$ by the strong continuity of the action of $G$ on $F$. So

$$
\varphi(\exp X)=\int_{0}^{1} \exp (t X) \cdot d \varphi(X) d t
$$

Since clearly $X \mapsto d \varphi(X)$ is linear it suffices to prove that $X \mapsto \int_{0}^{1} \exp (t X) \cdot F d t$ is continuous from $g$ into $F$ for any fixed $F \in F$. But that is a consequence of the fact that the integrand depends continuously on the parameter $X \in g$.

This proves the first half of the proposition.

Assume now, conversely, that $\varphi: G \rightarrow F$ is a continuous map that satisfies (6). Let $d \mu$ be a left Haar measure on $G$, and let $f \in C^{\infty}(G)$ be a real-valued function with compact support and $\int_{G} f d \mu=1$.

We multiply the identity (6) with $f(h)$, integrate with respect to $d \mu(h)$ and find

$$
\int_{G} f(h) \varphi(g h) d \mu(h)=\varphi(g)+g \cdot \int_{G} f(h) \varphi(h) d \mu(h)
$$

The last term is continuous by the assumption on the action of $G$ on $F$, so it suffices to prove that $g \mapsto \int_{G} f\left(g^{-1} h\right) \varphi(h) d \mu(h)$ is continuous. But the integrand clearly depends continuously on $(g, h) \in G \times G$.

LEMMA 2. If $F$ is quasi-complete and if $G$ is compact then $H^{1}(G, F)=0$.

PrOOF. Let $\varphi$ be an F-cocycle. It is then continuous. Upon integrating (6) over $G$ with respect to $d \mu(h)$ where $d \mu$ is the normalized Haar measure on $G$, we find that $\varphi$ is an F-coboundary with $F=-\int_{G} \varphi(h) d \mu(h)$. 
THEOREM 2. Let $G$ be a connected Lie group acting weakly $C^{\infty}$ 'ly on a quasi-complete, locally convex space $F$. Let us furthermore assume that $H^{1}(G)=0$ (de Rham cohomology). Then the linear operator $I: H^{1}(G, F) \rightarrow H^{1}(g, F)$ defined above is an isomorphism onto.

REMARK. Theorem 2 is a generalization to infinite-dimensional vector spaces of a result due to van Est [6]. It should be mentioned that G. Hochschild and G. D. Mostow [8] also have studied the case of an infinite-dimensional module.

EXAMPLE 2. The assumption $H^{1}(G)=0$ is essential as the following example shows:

Let the group $G=\operatorname{SL}(2, \mathbf{R})$ act on the upper half plane $P$ as follows:

$$
\left(\begin{array}{ll}
a & b \\
c & d
\end{array}\right) \cdot z=\frac{a z+b}{c z+d} \text { for }\left(\begin{array}{ll}
a & b \\
c & d
\end{array}\right) \in G, z \in P .
$$

It is well known that $G$ is a connected, simple group with $H^{1}(G) \cong \mathbf{R}$.

The isotropy group of $i \in P$ is the group of rotations $S O(2)$, so $P$ is diffeomorphic to $G / S O(2)$.

By brute calculations it can be shown that $H^{1}\left(g, C^{\infty}(P)\right) \cong \mathrm{R}$ and that $H^{1}\left(G, C^{\infty}(P)\right)=0$.

Proof of Theorem 2. The injectivity of $I$ is the content of Proposition 1 above, so it is left to prove that $I$ is surjective.

Let therefore $\Phi: g \rightarrow F$ be an arbitrary cocycle. Partly motivated by (8) we will for each $u \in F^{\prime}$ study the map $\omega^{u}: T_{g} G \rightarrow R$, defined by

$$
\left\langle\omega^{u}, X_{g}\right\rangle:=\langle u, g \cdot \Phi(X)\rangle \text { for } g \in G, X \in g \text {. }
$$

For the sake of clearness the rest of the proof is divided into a couple of lemmas.

LEMMA 3. $\omega^{u}$ is a closed differential 1-form on $G$, and

$$
L\left(g^{-1}\right)^{*} \omega^{u}=\omega^{g \cdot u} \quad \text { for all } g \in G, u \in F^{\prime} .
$$

Proof. It is obvious from the very definition of $\omega^{u}$ that it is a differential 1 -form on $G$. To prove it is closed it suffices to show that $d \omega^{u}\left(X_{g}, Y_{g}\right)=0$ for all $X, Y \in g, g \in G$. Now, by a well-known formula and (5):

$$
\begin{aligned}
d \omega^{u}\left(X_{g}, Y_{g}\right)= & X_{g}\left(\omega^{u}(Y .)\right)-Y_{g}\left(\omega^{u}(X .)\right)-\omega_{g}^{u}\left([X, Y]_{g}\right) \\
= & X_{g}(h \mapsto\langle u, h \cdot \Phi(Y)\rangle)-Y_{g}(h \mapsto\langle u, h \cdot \Phi(X)\rangle) \\
& -\langle u, g \cdot \Phi([X, Y])\rangle \\
= & \langle u, g \cdot(X \cdot \Phi(Y))\rangle-\langle u, g \cdot(Y \cdot \Phi(X))\rangle-\langle u, g \cdot \Phi([X, Y])\rangle \\
= & \langle u, g \cdot\{X \cdot \Phi(Y)-Y \cdot \Phi(X)-\Phi([X, Y])\}\rangle=0
\end{aligned}
$$

since $\Phi$ by assumption is a cocycle. 
The rest of the proof is a simple manipulation with the symbols involved:

$$
\begin{aligned}
\left(L\left(g^{-1}\right)^{*} \omega^{u}\right)_{h}\left(X_{h}\right) & =\omega_{g^{-1} h^{u}}\left(L\left(g^{-1}\right)_{*} X_{h}\right)=\omega_{g^{-1} h}^{u}\left(X_{g^{-1} h}\right) \\
& =\left\langle u, g^{-1} h \cdot \Phi(X)\right\rangle=\langle g \cdot u, h \cdot \Phi(X)\rangle=\omega^{g \cdot u}\left(X_{h}\right) .
\end{aligned}
$$

According to Lemma $3 \omega^{u}$ is a closed 1 -form on $G$, so by the assumption $H^{1}(G)=0$ there exists a function $I(\cdot, u) \in C^{\infty}(G)$ such that $\omega^{u}=d I(\cdot, u)$. The function is unique modulo a constant which we fix by requiring that $I(e, u)$ $=0$. Then

$$
I(g, u)=\int_{\gamma} \omega^{u}
$$

if $\gamma:[a, b] \rightarrow G$ is any differe:tiable curve in $G$ with $\gamma(a)=e$ and $\gamma(b)=g$.

\section{LEMMA 4.}

$$
I(g h, u)=I(g, u)+I\left(h, g^{-1} \cdot u\right) \quad \text { for all } g, h \in G, u \in F^{\prime} .
$$

Proof. (10) results by a simple application of Lemma 3:

$$
\begin{aligned}
I(g h, u) & =\int_{e}^{g h} \omega^{u}=\int_{e}^{g} \omega^{u}+\int_{g}^{g h} \omega^{u}=I(g, u)+\int_{e}^{h} L(g)^{*} \omega^{u} \\
& =I(g, u)+\int_{e}^{h} \omega^{g^{-1} \cdot u}=I(g, u)+I\left(h, g^{-1} \cdot u\right) .
\end{aligned}
$$

Lemma 5. There exists an F-cocycle $\varphi: G \rightarrow F$ such that

$$
I(g, u)=\langle u, \varphi(g)\rangle \text { for all } g \in G, u \in F^{\prime} .
$$

Proof. We first claim that to each $g \in G$ there exists an element $\varphi(g) \in F$ such that $I(g, u)=\langle u, \varphi(g)\rangle$ for all $u \in F^{\prime}$.

Since any neighbourhood of $e$ in $G$ generates $G$, it follows easily via (10) that it suffices to prove the claim for $g$ close to $e \in G$, so that we may assume $g=\exp X$ for some $X \in g$. Now,

$$
\begin{aligned}
I(g, u) & =\int_{e}^{g} \omega^{u}=\int_{0}^{1} \omega_{\exp (t X)}^{u}\left(X_{\exp (t X)}\right) d t \\
& =\int_{0}^{1}\langle u, \exp (t X) \cdot \Phi(X)\rangle d t=\left\langle u, \int_{0}^{1} \exp (t X) \cdot \Phi(X) d t\right\rangle
\end{aligned}
$$

which proves the claim.

(6) is simply (10) rewritten. $\varphi$ is obviously weakly $C^{\infty}$ since $\langle u, \varphi(\cdot)\rangle=$ $I(\cdot, u) \in C^{\infty}(G)$, so $\varphi$ is an F-cocycle.

Now,

$$
X\langle u, \varphi(\cdot)\rangle=X I(\cdot, u)=d I(\cdot, u)(X)=\left\langle\omega^{u}, X\right\rangle=\langle u, \Phi(X)\rangle,
$$

showing that $d \varphi(X)=\Phi(X)$ for all $x \in g$. This finishes the proof of Theorem 2 . 
Corollary 1. Let $G$ be a connected, compact and semisimple Lie group acting weakly differentiably on a quasi-complete space $F$. Then $H^{1}(g, F)=0$.

Proof. It is well known that $H^{1}(G)=0$ when $G$ is a compact semisimple Lie group (see, for example, [4, Theorem 21.1, p. 114]). The corollary thus follows from Theorem 2 and Lemma 2.

Corollary 2. Let $G$ be a connected, compact, semisimple Lie group acting as a Lie transformation group on a $C^{\infty}$ manifold $M$. Let $F$ be a closed $G$-invariant subspace of $C^{\infty}(M)$. Then $H^{1}(g, F)=0$.

Remark. The action of $G$ on $C^{\infty}(M)$ is described in Example 1.

Proof. It is well known that $C^{\infty}(M)$ is a Montel space, hence quasi-complete. $F$ is then also quasi-complete as a closed subspace. The result is thus immediate from Corollary 1 as soon as we have checked that $G$ acts differentiably on $F$ or rather just that it does in the weakened form indicated in the remark after Definition 3. Here we may as an invariant, separating subset of $F^{\prime}$ take the evaluations at points of $M$.

COROLlARY 3. Let $G$ be a connected Lie group, acting as a Lie transformation group on a $C^{\infty}$ manifold $M$. Assume $H^{1}(G)=0$, and that there exists an embedded submanifold $M^{\prime}$ of $M$ such that the map $\left(g, m^{\prime}\right) \mapsto g \cdot m^{\prime}$ is a diffeomorphism of $G \times M^{\prime}$ onto $M$. Then $H^{1}\left(G, C^{\infty}(M)\right)=0$ and $H^{1}\left(g, C^{\infty}(M)\right)=0$.

Proof. It suffices to prove that $H^{1}\left(G, C^{\infty}(M)\right)=0$, so let $\varphi: G \rightarrow C^{\infty}(M)$ be a cocycle. Define $F: M=G \times M^{\prime} \rightarrow \mathrm{R}$ by

$$
F\left(g m^{\prime}\right):=\varphi\left(g^{-1}\right)\left(m^{\prime}\right) \text { for } g \in G, m^{\prime} \in M^{\prime} .
$$

Then by an easy calculation, $\varphi(g)=g \cdot F-F$, so it is left to show that $F \in C^{\infty}(M)$, or equivalently, that the map $\left(g, m^{\prime}\right) \mapsto \varphi(g)\left(m^{\prime}\right)$ belongs to $C^{\infty}(M)$. By (8) we know that

$$
X_{g}\left[\varphi()\left(m^{\prime}\right)\right]=\left(g \cdot \varphi_{X}\right)\left(m^{\prime}\right)=\varphi_{X}\left(g^{-1} \cdot m^{\prime}\right)
$$

so that $\left(g, m^{\prime}\right) \mapsto X_{g}\left[\varphi(\cdot)\left(m^{\prime}\right)\right]$ is in $C^{\infty}(M)$.

By the cocycle condition (6) we need only check differentiability for $g$ close to the identity, i.e. that

$$
\left[\left(X, m^{\prime}\right) \mapsto \varphi(\exp X)\left(m^{\prime}\right)\right] \in C^{\infty}\left(g \times M^{\prime}\right)
$$

Let now $\left\{X_{1}, \ldots, X_{n}\right\}$ be a basis of $g$. Then 


$$
\begin{aligned}
\varphi(\exp X)\left(m^{\prime}\right) & \left.=\varphi\left(\exp \sum_{j} \lambda_{j} X_{j}\right)\right)\left(m^{\prime}\right) \\
& =\int_{0}^{1} \frac{d}{d t} \varphi\left(\exp \left(t \sum_{j} \lambda_{j} X_{j}\right)\right)\left(m^{\prime}\right) d t \\
& =\int_{0}^{1} X_{\exp (t X)}\left[\varphi(\cdot)\left(m^{\prime}\right)\right] d t \\
& =\int_{0}^{1} \varphi_{X}\left(\exp (-t X) \cdot m^{\prime}\right) d t \\
& =\sum_{j} \lambda_{j} \int_{0}^{1} \varphi_{X_{j}}\left(\exp (-t X) \cdot m^{\prime}\right) d t
\end{aligned}
$$

so it suffices to prove that

$$
\left(\lambda_{1}, \ldots, \lambda_{n}, m^{\prime}\right) \mapsto \int_{0}^{1} H\left(\exp \left(t \sum_{j} \lambda_{j} X_{j}\right) \cdot m^{\prime}\right) d t
$$

belongs to $C^{\infty}\left(\mathrm{R}^{n} \times M^{\prime}\right)$ for each fixed $H \in C^{\infty}(M)$. But the map $t \mapsto H_{t}$, where

$$
H_{t}\left(\lambda_{1}, \ldots, \lambda_{n}, m^{\prime}\right):=H\left(\exp \left(t \sum_{j} \lambda_{j} X_{j}\right) \cdot m^{\prime}\right),
$$

is clearly continuous from $[0,1]$ into $C^{\infty}\left(\mathrm{R}^{n} \times M^{\prime}\right)$ so the result follows from well-known facts about integration of vector valued functions.

EXAMPLE 3. Let $G$ act on $M:=G \times G \times \cdots \times G$ ( $r$ factors) by

$$
g \cdot\left(g_{1}, g_{2}, \ldots, g_{r}\right):=\left(g g_{1}, g g_{2}, \ldots, g g_{r}\right) .
$$

If $H^{1}(G)=0$ then all the hypotheses of Corollary 3 are satisfied with

$$
M^{\prime}=\{e\} \times G \times \cdots \times G,
$$

so $H^{1}\left(g, C^{\infty}(M)\right)=0$.

V. Use of the Levi decomposition. We proceed by studying how Leutwyler's three types of groups enter in the cohomology framework, and we use the results to prove (a generalized version of) Leutwyler's theorem.

Any Lie algebra $g$ is, by Levi's theorem $[9$, p. 91], a semidirect sum $g=n$ $t_{s}, 5$ of a solvable ideal $n$ and a semisimple subalgebra 5 . Leutwyler's type (c) group corresponds to a Levi decomposition of $\mathrm{g}$ : Indeed, the Lie groups that have compact covering group are exactly the compact semisimple ones (that is a consequence of [7, Theorem II. 6.9 and Proposition II. 6.6]).

Let the Lie algebra $g$ be a semidirect sum

$$
g=n+s
$$

of an ideal $n$ and a subalgebra $\bar{G}$ of $g$. So we have a short exact sequence of Lie algebras, $0 \rightarrow n \rightarrow g \rightarrow \mathfrak{G} \rightarrow 0$. The Hochschild-Serre spectral sequence of homological algebra (see $[2$, p. 351$]$ ) associates by purely algebraic manipulations to 
each such short exact sequence a long exact sequence of cohomology spaces

with obvious actions.

$$
\begin{aligned}
0 \rightarrow H^{1}\left(\xi, H^{0}\left(\mathrm{n}, C^{\infty}(M)\right)\right) & \rightarrow H^{1}\left(\mathrm{~g}, C^{\infty}(M)\right) \\
& \rightarrow H^{0}\left(\xi, H^{1}\left(\mathrm{n}, C^{\infty}(M)\right)\right) \rightarrow \cdots
\end{aligned}
$$

If Leutwyler's problem can be solved for $n$, that is according to Theorem 1 if $H^{1}\left(n, C^{\infty}(M)\right)=(0)$, then the long exact sequence (13) tells us that

$$
H^{1}\left(g, C^{\infty}(M)\right) \cong H^{1}\left(\mathfrak{g}, H^{0}\left(\mathrm{n}, C^{\infty}(M)\right)\right) \text {. }
$$

So in that case the problem boils down to the Leutwyler problem for 5 , except for the fact that $C^{\infty}(M)$ should be replaced by the subspace $H^{0}\left(n, C^{\infty}(M)\right)$.

Therefore we now find a criterion which ensures $H^{1}\left(\mathfrak{n}, C^{\infty}(M)\right)=0$. It is very close to Corollary 3.

Let $N$ be the analytic subgroup of $G$ corresponding to $n$. The situation may here be very complicated even in the case $N=\mathbf{R}$ (a dynamical system) where the compatibility conditions (3) trivially are satisfied. Note, for example, that Leutwyler's problem cannot be solved if $X_{1}^{*}$ vanishes at a point of $M$. Whether $X_{1}^{*} C^{\infty}(M)=C^{\infty}(M)$ depends on the behaviour of the integral curves of $X_{1}^{*}$ (see $[5$, pp. 212ff]).

In applications to physics, $n$ will often be the ideal in $g$ that corresponds to the translations. In such cases the map $X \mapsto X_{p}^{*}$ is injective from $n$ into $T_{p} M$ for each $p \in M$. An equivalent way of stating this is that the map $g \mapsto g \cdot p$ of $N$ into $M$ should be an immersion for each fixed $p \in M$. With $N_{p}:=\{g \in N \mid g \cdot p=p\}$, a third equivalent formulation is that $N_{p}$ is a discrete subgroup of $N$ for each $p \in M$.

THEOREM 3. If

(1) there exists an embedded submanifold $M^{\prime}$ of $M$ such that $(n, p) \mapsto n \cdot p$ is a regular map of $N \times M^{\prime}$ onto $M$, and

(2) each orbit $N \cdot p$ intersects $M^{\prime}$ in exactly one point, and

(3) $H^{1}\left(N / N_{p}\right)=\{0\}$ for each $p \in M$ (de Rham cohomology), then $H^{1}\left(n, C^{\infty}(M)\right)=\{0\}$.

Proof. Let $\Phi: n \rightarrow C^{\infty}(M)$ be any cocycle.

Any orbit $O=N \cdot p$ is, by assumption (1), an immersed submanifold of $M$ under the obvious immersion of $N / N_{p}$ onto $O$.

Let $\omega_{O}: T_{p} O \rightarrow \mathrm{R}$ be the linear map

$$
\omega_{O}\left(X_{p}^{*}\right)=\Phi(X)(p) \text { for } X \in \mathrm{n} .
$$

Now $\omega_{O}$ is a smooth differential 1 -form on the manifold $O$. We compute its exterior differential: If $X, Y \in n$, then 


$$
\begin{aligned}
d \omega_{O}\left(X^{*}, Y^{*}\right) & =X^{*} \omega_{O}\left(Y^{*}\right)-Y^{*} \omega_{O}\left(X^{*}\right)-\omega_{O}\left(\left[X^{*}, Y^{*}\right]\right) \\
& =X^{*} \Phi(Y)-Y^{*} \Phi(X)-\Phi([X, Y])=0,
\end{aligned}
$$

since $\Phi$ is a cocycle. Since $H^{1}(O)=H^{1}\left(N / N_{p}\right)=0$, by assumption (3) there is a function $f_{O} \in C^{\infty}(O)$ such that $\omega_{O}=d f_{O}$. It is unique modulo an additive constant.

Let $F$ be the function defined by $F l_{O}=f_{O}$ for all orbits $O$. If the additive constants can be chosen in such a way that $F \in C^{\infty}(M)$, then $\Phi$ is a coboundary, viz. $\Phi=\delta F$ :

$$
\begin{aligned}
{[(\delta F(X))](p) } & =\left(X^{*} F\right)(p)=X_{p}^{*} F=X_{p}^{*} f_{N \cdot p} \\
& =d f_{N \cdot p}\left(X_{p}^{*}\right)=\omega_{N \cdot p}\left(X_{p}^{*}\right)=\Phi(X)(p) .
\end{aligned}
$$

Hence Leutwyler's problem can be solved. It is thus left to show that $f_{O}$ may be chosen so that $F \in C^{\infty}(M)$. Let us normalize the $f_{O}$ by requiring that $\left.F\right|_{M^{\prime}}=0$.

It now suffices to show that $H\left(n, p^{\prime}\right):=F\left(n p^{\prime}\right)$ is a $C^{\infty}$ function on $N \times M^{\prime}$, because $\left(n, p^{\prime}\right) \mapsto n p^{\prime}$ is regular. But $H\left(\cdot, p^{\prime}\right)$ is that solution to

$$
\left(X_{A}\right)_{n}^{r} H\left(\cdot, p^{\prime}\right)=-\Phi\left(X_{A}\right)\left(n p^{\prime}\right), \quad A=1, \ldots, n,
$$

that satisfies $H\left(e, p^{\prime}\right)=0$. Well-known results about the dependence of solutions to differential equations now ensure the result.

Remarks. (a) Example 3 with $G=N$ is an easy consequence of Theorem 3.

(b) It can be shown that $H^{1}(N)=0$ in Theorem 3 so the theorem is more restrictive about the group than Corollary 3 which, on the other hand, assumes more about the manifold.

(c) Condition (3) is probably rather restrictive. It follows for example from [16, Corollary 7.28, p. 123] that $N_{p}$ cannot be a lattice in case $N$ is a nilpotent simply connected group.

COROLlaRY 4. Let $\mathrm{g}=\mathfrak{n}+_{s} \mathfrak{g}$ be a semidirect sum where $\bar{g}$ is semisimple. If

(a) the map $X \mapsto X_{p}^{*}$ is an isomorphism of $n$ onto $T_{p} M$ for each $p \in M$,

(b) $H^{1}(M)=0$, then $H^{1}\left(g, C^{\infty}(M)\right)=0$.

Proof. The hypotheses of the theorem are clearly satisfied since $M$ in this case is transitive under the action of $N$. By the earlier formula (13) it suffices to prove that $H^{1}\left(\xi, H^{0}\left(n, C^{\infty}(M)\right)=0\right.$. But $H^{0}\left(n, C^{\infty}(M)\right)$ reduces to the constant functions by assumption (a) of the corollary, so $H^{0}\left(n, C^{\infty}(M) \approx \mathbf{R}\right.$.

The corollary is now a consequence of Whitehead's 1st lemma (see [9]).

EXAMPLE 4. The hypotheses of the corollary are satisfied for the Poincaré group $P_{+}^{\uparrow}:=\mathbf{R}^{4} \times{ }_{s} O(3,1)$ acting on $\mathbf{R}^{4}$.

THEOREM 4. Let us assume $g=n+{ }_{s} 5$, where $n$ is an ideal in $g$ with $H^{1}\left(\mathrm{n}, C^{\infty}(M)\right)=0$ and where $\mathrm{g}$ is a compact semisimple subalgebra of $\mathrm{g}$. Then $H^{1}\left(g, C^{\infty}(M)\right)=\{0\}$. 
Proof. Going back to (14) we see it is enough to show that

$$
H^{1}\left(\xi, H^{0}\left(n, C^{\infty}(M)\right)=0 .\right.
$$

But that is a particular case of Corollary 2 .

COROllaRY 5. Leutwyler's theorem (cf. §II).

ProOF.

Case (a). We have already remarked in the beginning of this section that the groups with compact covering group precisely are the compact, semisimple groups, so the case is taken care of by Corollary 2 .

Case (b). Since the translation group $N$ obviously has a cross section-for example the subspace orthogonal to all the translations from $N$-it follows by an appeal to Corollary 3 that $H^{1}\left(n, C^{\infty}(M)\right)=0$.

Case (c). Here we just combine Case (b) and Theorem 4.

VI. The case of transitive action. If we are given just a subgroup of $G$, not necessarily a semidirect decomposition of $g$, then we can still in some cases manage to solve Leutwyler's problem. The present section is essentially devoted to the case of a product of transitive $G$-spaces. The idea is the obvious one: We first solve Leutwyler's problem for $G$ acting on itself, and then see whether the solution can be chosen invariant under a given subgroup $K$, so that Leutwyler's problem can be solved for $G$ acting on $G / K$.

Let us first note a purely algebraic lemma. $F$ will be a g-module, $F_{0}$ a g-submodule. We may ask whether the generalized Leutwyler problem can be solved for $F_{0}$ if it can for $F$, i.e. whether $H^{1}\left(g, F_{0}\right)=0$ when $H^{1}(g, F)=0$.

Lemma 6. If there exists a projection $P$ of $F$ onto $F_{0}$ such that

$$
P(X \cdot F)=X \cdot P(F) \text { for all } X \in g, F \in F,
$$

then $H^{1}(g, F)=0$ implies $H^{1}\left(g, F_{0}\right)=0$.

Proof. Any cocycle $\Phi: g \rightarrow F_{0}$ may be viewed as a cocycle with values in $F$, and so by assumption there exists an $F \in F$ such that $\Phi(X)=X \cdot F$ for all $X \in$ g. Applying $P$ to both sides of this identity we find by (14) that

$$
\Phi(X)=X \cdot P F,
$$

so $\Phi$ is the coboundary of $P F \in F_{0}$.

From now on let the situation be as in $\S I V: F$ is a locally convex topological vector space on which there is given a strongly continuous, weakly $C^{\infty}$ representation of a connected Lie group $G$, etc. In this section we will assume that there, in addition, is given a representation $\rho$ of a group $K$ on $F$ by continuous linear operators such that 


$$
g \cdot \rho(k)(F)=\rho(k)(g \cdot F) \text { for all } g \in G, k \in K, F \in F \text {. }
$$

Then

$$
F_{K}:=\{F \in F \mid \rho(k) F=F \text { for all } k \in K\}
$$

is a closed subspace of $F$, invariant under the actions of $G$ and $g$.

PRoposition 3. If $F$ is a quasi-complete, locally convex topological vector space, if $K$ is a compact group, and if the map $(k, F) \mapsto \rho(k)(F)$ on $K \times F$ into $F$ is continuous, then $H^{1}(g, F)=0$ implies $H^{1}\left(g, F_{K}\right)=0$.

Proof. It follows from well-known properties of integration of vector valued functions that

$$
P:=\int_{K} \rho(k) d \mu(k),
$$

where $d \mu$ is the normalized Haar measure on $K$, is a continuous projection of $F$ onto $F_{K}$. Obviously

$$
P(g \cdot F)=g \cdot P(F) \text { for all } g \in G, F \in F,
$$

from which we get (14) by differentiation, so that the result is trivial by Lemma 6 .

REMARK. If $F$, in addition to being quasi-complete, is barrelled, then strong continuity of $\rho$ suffices in Proposition 3, because the map $(k, F) \mapsto \rho(k) F$ by Banach-Steinhaus then automatically is continuous.

We will now specialize the above results to the case of a Lie transformation group.

Let $G$ be a connected Lie group with $H^{1}(G)=0$, acting as a Lie transformation group on a manifold $M$. Then $M^{\prime}:=G \times M$ is a $G$-space under the action $g \cdot\left(g_{1}, m\right)=\left(g g_{1}, g m\right)$ for $g, g_{1} \in G, m \in M$, and $H^{1}\left(g, C^{\infty}\left(M^{\prime}\right)\right)=0$ according to Corollary 3.

Let $K$ be a compact subgroup of $G$ and define a representation $\rho$ of $K$ on $F=C^{\infty}\left(M^{\prime}\right)$ by

$$
[\rho(k) F](g, m):=F\left(g k^{-1}, m\right) \text { for all } F \in C^{\infty}(M), g \in G, m \in M, k \in K .
$$

Then $\rho$ satisfies all the hypotheses of Proposition 3, so we conclude that $H^{1}\left(g, F_{K}\right)=0$. But here

$$
\begin{aligned}
F_{K} & =\left\{F \in C^{\infty}\left(M^{\prime}\right) \mid F(g k, m)=F(g, m) \text { for all } g \in G, k \in K, m \in M\right\} \\
& =C^{\infty}(G / K \times M),
\end{aligned}
$$

so we have proved

THEOREM 5. Let $G$ be a connected Lie group with $H^{1}(G)=0$ acting as a Lie transformation group on a manifold $M$. Let $K$ be a compact subgroup of $G$.

Then Leutwyler's problem for $G$ acting on $G / K \times M$ has solutions. 
COROLlary 6. Let $G$ be a connected Lie group with $H^{1}(G)=0$, and let $K_{1}, \ldots, K_{r}$ be closed subgroups.

Then Leutwyler's problem for $G$ acting on $G / K_{1} \times \cdots \times G / K_{r}$ has solutions if one of the groups $K_{1}, \ldots, K_{r}$ is compact.

REMARK. Leutwyler's result about "inhomogeneous compact groups" of the form $\mathbf{R}^{n} \times{ }_{s} H$, acting on $\mathbf{R}^{n} \times \cdots \times \mathbf{R}^{n}$, is an obvious consequence of Corollary 6.

If the subgroup $K$ from Proposition 4 is not compact, our procedure breaks down. But we can still obtain some results if we use the special form of the action.

Let us first of all agree on the notation: $G$ is a connected Lie group with $H^{1}(G)=0$ acting as a Lie transformation group on a manifold $M$, and $K$ is a closed subgroup. Let $\pi: G \times M \rightarrow G / K \times M$ be the projection, and let $\bar{X}\left(X^{*}\right)$ be the vector field on $G \times M(G / K \times M)$ corresponding to $X \in g$ under the action of $G$.

We want to see whether Leutwyler's problem for $G$ acting on $G / K \times M$ has solutions so we let $\Phi: g \rightarrow C^{\infty}(G / K \times M)$ be an arbitrary cocycle. Then $X \mapsto$ $\Phi(X) \circ \pi$ is a cocycle w.r.t. $C^{\infty}(G \times M)$, so according to Corollary 3 there exists $H \in C^{\infty}(G \times M)$ such that

$$
\Phi(X) \circ \pi=\bar{X} H \text { for all } X \in g .
$$

Furthermore it follows from (16) that $H$ is unique modulo functions that are constant on the orbits of the action of $G$ on $G \times M$, so we may and will normalize $H$ by requiring $H(e, m)=0$ for all $m \in M$.

An easy computation shows that the function $\rho(k) H$ for fixed $k \in K$ also satisfies (16) and, hence, differs from $H$ by at most a function that is constant on the orbits. That function is fixed by the normalization, which gives us

$$
H(g k, m)=H(g, m)+H\left(k, g^{-1} m\right) \text { for all } g \in G, k \in K, m \in M \text {. }
$$

THEOREM 6. Let $H^{1}(G)=0$. Leutwyler's problem for $G$ acting on $G / K \times M$ has solutions if and only if every solution $H \in C^{\infty}(G \times M)$ of $(17)$ on $K \times M$ is of the form $H(k, m)=b\left(k^{-1} m\right)-b(m)$ for some function $b \in C^{\infty}(M)$.

Proof. Let first $\Phi$ (a cocycle) and $H$ be as above. If $H$ is of the form stated in the theorem, then an easy computation shows that

$$
H_{1}(g, m):=H(g, m)-b\left(g^{-1} m\right)
$$

depends only on the coset $g K$ and $m$, so that $H_{1}$ can be viewed as a function on $G / K \times M$. Replacing $H$ with $H_{1}$, we find $\Phi=\delta H_{1}$.

Assume conversely that Leutwyler's problem has solutions. If $H \in C^{\infty}(G \times M)$ satisfies (17), then $\bar{X} H$ for any $X \in g$ only depends on the cosets and may, hence, 
be written in the form

$$
\bar{X} H=\Phi(X) \circ \pi \quad \text { where } \Phi(X) \in C^{\infty}(G / K \times M) .
$$

Obviously $\Phi$ is a cocycle, so by assumption there is an $F \in C^{\infty}(G / K \times M)$ such that $\Phi=\delta F$. Now

$$
\bar{X} H=\Phi(X) \circ \pi=\left(X^{*} F\right) \circ \pi=\bar{X}(F \circ \pi),
$$

so that $H-F \circ \pi$ is constant on the orbits and, hence, of the form

$$
H(g, m)-F(g K, m)=b\left(g^{-1} m\right) \text { for some } b \in C^{\infty}(M) .
$$

Putting $g=e$ here we get $F(K, m)=-b(m)$ and so

as desired.

$$
H(k, m)=b\left(k^{-1} m\right)-b(m)
$$

REMARK. Part of the theorem may easily be stated in cohomological terms. With the notation of Varadarajan [17, pp. 27ff]:

Leutwyler's problem has solutions if every smooth $(K, M, \mathbf{R})$-cocycle is a smooth coboundary.

We are mainly interested in $r>1$, since the case $r=1$, in the application we know of, corresponds to the situation where there is just one particle in the universe! For the sake of completeness we mention how the set-up above simplifies in the case $r=1$, where we put $K=K_{1}$.

(17) reduces to

$$
H(g k)=H(g)+H(k) \text { for } g \in G, k \in K .
$$

Let $\operatorname{Hom}(G, \mathbf{R})$ denote the set of continuous homomorphisms of the group $G$ into $(\mathbf{R},+)$.

THEOREM 7. Let $H^{1}(G)=0$. Leutwyler's problem for $G$ acting on $G / K$ has solutions if and only if every solution $H \in C^{\infty}(G)$ of $(18)$ reduces to 0 on $K$. In particular, Leutwyler's problem has solutions if $\operatorname{Hom}(K, \mathbf{R})=\{0\}$.

COROLlaRY 7. Leutwyler's problem has solutions if

(a) $K$ is compact, or

(b) $K$ is semisimple with at most finitely many components, or

(c) $K=N \times{ }_{s} H$, where $\operatorname{Hom}(H, \mathbf{R})=\{0\}$ and $\left\{f \in \operatorname{Hom}(N, \mathbf{R}) \mid f\left(h^{-1} n h\right)\right.$ $=f(n)$ for $n \in N, h \in H\}=(0)$.

REMARK. The standing assumption in this section, $H^{1}(G)=0$, cannot be relaxed, in general, not even in the case $r=1$. In the case of $\operatorname{SL}(2, R)$ acting on $P:=\mathrm{SL}(2, \mathrm{R}) / S O(2)$, one finds $H^{1}\left(\mathrm{~g}, C^{\infty}(P)\right) \cong \mathrm{R}$ (cf. Example 2 ).

EXAMPLE 5. Example 4 is a consequence of Theorem 7, because $R^{4}=$ $P_{+}^{\uparrow} / O(3,1)$ and $O(3,1)$ is semisimple and $H^{1}\left(P_{+}^{\uparrow}\right)=0$. 
EXAMPLE 6. Let $G=\mathrm{R}^{3} \times{ }_{s} S O(3)$ be the group of orientation preserving rigid motions of $\mathbf{R}^{3}$ with the action

$$
(\vec{v}, A) \overrightarrow{(x)}=\overrightarrow{A x}+\vec{v} \quad \text { for }(\vec{v}, A) \in G, \vec{x} \in \mathbf{R}^{3}
$$

Extend this action diagonally to an action of $G$ on

$$
\left(\mathbf{R}^{3}\right)^{r}=G / S O(3) \times \cdots \times G / S O(3) \quad(r \text { factors }) .
$$

By Corollary 6 we get $H^{1}\left(g, C^{\infty}\left(\mathrm{R}^{3 n}\right)\right)=0$.

EXAMPLE 7. Let $G=\mathbf{R}^{4} \times{ }_{s} I_{0}\left(\mathbf{R}^{3}\right)$ be the Galilei group. Here $I_{0}\left(\mathbf{R}^{3}\right)=$ $\mathbf{R}^{3} \times{ }_{s} S O(3)$ is the group of orientation preserving isometries of $\mathbf{R}^{3}$. The group $G$ acts transitively on $\mathrm{R}^{4}$ with isotropy group $I_{0}\left(\mathrm{R}^{3}\right)$, so we may view $\mathrm{R}^{4}$ as $G / I_{0}\left(R^{3}\right)$.

It is a consequence of Corollary 7 that Leutwyler's problem has solutions in this case.

REMARK. The action of the Galilei group on $\mathbf{R}^{3 n} \times \mathbf{R}$ can be treated by help of the spectral sequence. In that way one easily regains Leutwyler's results about this case. We omit the discussion because the results are not new and because the proof involves the second Hochschild cohomology group that we have not introduced.

VII. Preliminaries on Hodge theory. Let $M$ be a Riemannian manifold. Let $\Omega^{r}$ $=\Omega^{r}(M)$ for $r=0,1, \ldots$ denote the real vector space of all differential $r$-forms on $M$ equipped with the usual Fréchet topology. Then both the exterior differential $d$ and the codifferential $\delta$ of Hodge theory are continuous linear operators.

From now on let $M$ be compact. Then $\Omega^{r}(M)$ can, by Hodge's theorem, be expressed as a direct sum

$$
\Omega^{r}=d \Omega^{r-1} \oplus \delta \Omega^{r+1} \oplus \Omega_{\text {harmonic }}^{r},
$$

where $\Omega_{\text {harmonic }}^{r}$ denotes the subspace of harmonic differential $r$-forms. The decomposition is orthogonal with respect to the $L^{2}$-product of differential forms. All three subspaces of $\Omega^{r}$ are closed. The projections on them are, of course, continuous with respect to the $L^{2}$-product and, hence (by the closed graph theorem), also continuous with respect to the usual Fréchet topologies above.

The following lemma is well known.

LEMMA 7. If $H^{1}(M)=0$ (de Rham cohomology), then the only harmonic 1 -form on $M$ is 0 .

In particular, if $M=G$ is a compact semisimple Lie group, then $\Omega_{\text {harmonic }}^{1}(G)=\{0\}$. 
Proposition 4. The maps $\delta: \Omega^{r} \rightarrow \delta \Omega^{r}$ and $d: \Omega^{r} \rightarrow d \Omega^{r}$ have continuous right inverses, say $\widetilde{\delta}: \delta \Omega^{r} \rightarrow \Omega^{r}$ and $\widetilde{d}: d \Omega^{r} \rightarrow \Omega^{r}$, with the following property:

If $L: \Omega^{0} \oplus \Omega^{1} \oplus \cdots \rightarrow \Omega^{0} \oplus \Omega^{1} \oplus \cdots$ is any linear graded map which commutes with $d$ and $\delta$, then it commutes with $\widetilde{\delta}$ and $\widetilde{d}$.

Proof. We will only treat the map $\delta$, because $d$ can be handled quite analogously.

Let us first note that $\delta \Omega^{r}$ as a closed subspace of the Fréchet space $\Omega^{r-1}$ itself is a Fréchet space. Similarly for $d \Omega^{r-1}$.

Now $\delta$ vanishes on the parts $\delta \Omega^{r+1}$ and $\Omega_{\text {harmonic }}^{r} \Omega^{r}=d \Omega^{r-1}+$ $\delta \Omega^{r+1}+\Omega_{\text {harmonic }}^{r}$, so to prove the existence of a right inverse $\tilde{\delta}: \delta \Omega^{r} \rightarrow d \Omega^{r-1}$ it suffices to check that $\delta: d \Omega^{r-1} \rightarrow \delta \Omega^{r} \subseteq \Omega^{r-1}$ is injective. $\widetilde{\delta}$ will automatically be continuous according to the closed graph theorem, since $\delta: d \Omega^{r-1} \rightarrow \delta \Omega^{r}$ is a continuous map between Fréchet spaces.

But if $\delta d \omega^{r-1}=0$ for some $\omega^{r-1} \in \Omega^{r-1}$, then

$$
\left\|d \omega^{r-1}\right\|^{2}=\left(d \omega^{r-1}, d \omega^{r-1}\right)=\left(\delta d \omega^{r-1}, \omega^{r-1}\right)=0 .
$$

The statement about $L$ is obvious with the above definition of $\widetilde{\delta}$.

Let $G$ be an $n$-dimensional Lie group with Lie algebra $g$. Let $(\cdot, \cdot)$ be an arbitrary inner product on $g$. Then we get a Riemannian structure $(\cdot, \cdot)$ on all of $G$ by left-translations, namely the one defined by

$$
\left(L(g)_{*} X, L(g)_{*} Y\right):=(X, Y) \text { for all } g \in G, X, Y \in g,
$$

so that we can apply the Hodge machinery described above.

LEMMA 8. For any $g \in G$ we have

$$
\begin{gathered}
L(g)^{*} *=* L(g)^{*}, \\
L(g) * \delta=\delta L(g)^{*}, \quad L(g) * \widetilde{\delta}=\widetilde{\delta} L(g)^{*} \text { and } L(g)^{*} \tilde{d}=\widetilde{d} L(g)^{*} .
\end{gathered}
$$

Proof. According to Proposition 4 the two last statements of (21) follow from the first, so we need only prove that one. For that it suffices to prove (20). That again follows if we check that $L(g)^{*} * \omega=* L(g)^{*} \omega$ for all $\omega \in \Omega^{r}$ of the form $\omega=\omega^{1} \wedge \cdots \wedge \omega^{r}$, where $\left\{\omega^{1}, \ldots, \omega^{n}\right\}$ are left-invariant 1-forms on $G$ such that $\left\{\omega_{e}^{1}, \ldots, \omega_{e}^{n}\right\}$ is a positively oriented orthonormal basis of $g^{*}$. Now,

$$
\begin{aligned}
L(g)^{*} *\left(\omega^{1} \wedge\right. & \left.\cdots \wedge \omega^{r}\right)=L(g)^{*} \omega^{r+1} \wedge \cdots \wedge \omega^{n} \\
& =\omega^{r+1} \wedge \cdots \wedge \omega^{n}=*\left(\omega^{1} \wedge \cdots \wedge \omega^{r}\right) \\
& =* L(g)^{*}\left(\omega^{1} \wedge \cdots \wedge \omega^{r}\right) .
\end{aligned}
$$

VIII. Vector valued forms.

Definition 5. Let $V$ and $F$ be real vector spaces. An F-valued $r$-form $\omega$ on $V$ is an alternating $r$-linear map $\omega: V \times V \times \cdots \times V$ ( $r$ factors $) \rightarrow F$. 
We let $\Lambda^{r}(V, F)$ denote the real vector space of $F$-valued $r$-forms on $V$, and let $\Lambda^{r}(V)$ be the space $\Lambda^{r}(V)=\Lambda^{r}(V, \mathbf{R})$.

LEMma 9. There is a canonical injection $I: \Lambda^{r}(V) \otimes F \rightarrow \Lambda^{r}(V, F)$, viz. the linear map determined by

$$
\begin{aligned}
& I(\omega \otimes f)\left(v_{1}, \ldots, v_{r}\right)=\omega\left(v_{1}, \ldots, v_{r}\right) f \\
& \qquad \text { for } \omega \in \Lambda^{r}(V), f \in F \text { and all } v_{1}, \ldots, v_{r} \in V .
\end{aligned}
$$

If $V$ is finite dimensional, then $I$ is a surjective isomorphism.

Proof. Elementary and left to the reader.

In the sequel, $V$ will always be finite dimensional, and we will identify $\Lambda^{r}(V) \otimes F$ and $\Lambda^{r}(V, F)$ as above without explicitly mentioning it each time.

If, in particular, $V$ is oriented and endowed with an inner product, then we have Hodge's star operator

$$
*: \Lambda^{r}(V) \rightarrow \Lambda^{n-r}(V), \text { where } n=\operatorname{dim} V .
$$

The operator

$$
* \otimes \mathrm{id}: \Lambda^{r}(V) \otimes F \rightarrow \Lambda^{n-r}(V) \otimes F
$$

will of convenience again be denoted by *. So

$$
*: \Lambda^{r}(V, F) \rightarrow \Lambda^{n-r}(V, F) \text {. }
$$

Another special case is the one where $V=g$ is a Lie algebra and where $F$ is a g-module. In this case we have a coboundary operator $d: \Lambda^{r}(V, F) \rightarrow$ $\Lambda^{r+1}(V, F)$, given by the standard formula

$$
\begin{aligned}
(d \Omega)\left(X^{1}, \ldots, X^{r+1}\right)= & \sum_{j=1}^{r+1}(-1)^{j+1} X^{j} \cdot \Omega\left(X^{1}, \ldots, \widehat{X^{j}}, \ldots, X^{r+1}\right) \\
& +\sum_{i<j}(-1)^{i+j} \Omega\left(\left[X^{i}, X^{i}\right], X^{1}, \ldots, \widehat{X^{i}}, \ldots, \widehat{X^{j}}, \ldots, X^{r+1}\right)
\end{aligned}
$$

for $\Omega \in \Lambda^{r}(V, F)$ and $X^{1}, \ldots, X^{r+1} \in g$.

If $g$, in addition, is equipped with an inner product, then we define, by help of Hodge's star operator, the codifferential

$$
\delta:=(-1)^{n r+n+1} * d *: \Lambda^{r}(g, F) \rightarrow \Lambda^{r-1}(g, F)
$$

and the "Laplace-Beltrami" operator

$$
\Delta:=d \delta+\delta d: \Lambda^{r}(g, F) \rightarrow \Lambda^{r}(g, F) .
$$

An $r$-form $\Omega \in \Lambda^{r}(g, F)$ is said to be harmonic in case $d \Omega=0$ and $\delta \Omega=0$.

Then, of course, $\Delta \Omega=0$. 
Let us now turn to the special case that we have studied earlier:

$F$ is a locally convex topological vector space. $G$ is a $L i e$ group and there is given a strongly continuous, weakly $C^{\infty}$ representation of $G$ on $F$. Then $F$ is, in particular, a g-module.

We shall see how the operators $d$ and $\delta$ on the differential forms of $G$ induce the corresponding operators $d$ and $\delta$ described above:

Let us for $\Omega \in \Lambda^{r}(g, F)$ and $u \in F^{\prime}$ define a differential $r$-form $\Omega^{u}$ on $G$ by $\Omega^{u}\left(X_{g}^{1}, \ldots, X_{g}^{r}\right):=\left\langle u, g \cdot \Omega\left(X^{1}, \ldots, X^{r}\right)\right\rangle$ for $g \in G, X^{1}, \ldots, X^{r} \in g$.

A small calculation now shows that

$$
d\left(\Omega^{u}\right)=(d \Omega)^{u} \quad \text { for all } \Omega \in \Lambda^{r}(g, F) \text { and } u \in F^{\prime},
$$

which provides us with an equivalent definition of $d$.

To get a similar characterization of $\delta$ we note the following lemma.

LEMma 10. If $\Omega \in \Lambda^{r}(g, F)$ and $u \in F^{\prime}$, then $*\left(\Omega^{u}\right)=(* \Omega)^{u}$.

Proof. An easy manipulation of the very definition of $\Omega^{u}$ shows that $L\left(g^{-1}\right) * \Omega^{u}=\Omega^{\cdot} u$ for all $g \in G$. That again implies easily that it suffices to prove the lemma at the identity, that is $*\left(\Omega^{u}\right)_{e}=(* \Omega)_{e}^{u}$. Since both sides are linear in $\Omega$ we may assume $\Omega$ is of the simple form $\Omega=\omega \otimes f \in \Lambda^{r}(g) \otimes F$. Then we find, by easy calculations,

$$
(* \Omega)_{e}^{u}=\langle u, f\rangle * \omega \text { and } *\left(\Omega_{e}^{u}\right)=*(\langle u, f\rangle \omega)=\langle u, f\rangle * \omega,
$$

which proves the lemma.

COROLlaRY 8. $\delta: \Lambda^{r}(g, F) \rightarrow \Lambda^{r-1}(g, F)$ may be defined by

$$
(\delta \Omega)^{u}=\delta\left(\Omega^{u}\right) \quad \text { for } \Omega \in \Lambda^{r}(g, F) \text { and } u \in F^{\prime} \text {. }
$$

IX. The generalized angular momentum first Helmholtz theorem. Let $F$ be a quasi-complete, locally convex topological vector space. Let us furthermore assume given a strongly continuous, weakly $C^{\infty}$ representation of a compact Lie group $G$ on $F$.

Theorem 8. Any linear map $\Phi: g \rightarrow F$ can in exactly one way be decomposed as

$$
\Phi=d F+\delta \Psi+\theta
$$

where $F \in F, \Psi \in \Lambda^{2}(g, F)$ and $\Theta$ is a harmonic map of $g$ into $F$.

If $g$ is semisimple, then $\Theta=0$.

REMARK. The individual terms $d F, \delta \Psi$ and $\Theta$ are uniquely determined, but $F$ and $\Psi$, of course, are not. 
REMARK. An example due to Keller shows that the assumption about $F$ being a $G$-module, in general, cannot be relaxed to $F$ being a g-module.

EXAMPLE 8. The Lie algebra $g$ of the circle group $G=S O(2)=\left\{e^{i \theta} \mid \theta \in R\right\}$ is spanned by $\partial / \partial \theta$ and, hence, can be identified with $\mathbf{R}$. We define a Riemannian structure on $S O(2)$ by requiring that $\partial / \partial \theta$ be a unit vector.

If we let $d \theta$ denote the 1 -form dual to $\partial / \partial \theta$, then it is easy to describe the Hodge star operator. Indeed

$$
* 1=d \theta \text { and } * d \theta=1 \text {. }
$$

A 1 -form $\omega=f(\theta) d \theta$ is harmonic if and only if $f$ is constant, and $\delta \omega=0$ if and only if $\int_{0}^{2 \pi} f(\theta) d \theta=0$.

Let

$$
\begin{aligned}
F_{0} & =\{F \in F \mid X \cdot F=0 \text { for all } X \in g\} \\
& =\{F \in F \mid g \cdot F=F \text { for all } g \in G\} .
\end{aligned}
$$

We find that a linear map $\Phi: g \rightarrow F$ is harmonic if and only if $\Phi(g) \subseteq F_{0}$.

The theorem above now implies that any linear map $\Phi: g \rightarrow F$ can be written in the form

$$
\Phi=d F+\Theta \text { where } F \in F \text { and } \Theta: g \rightarrow F_{0},
$$

or equivalently:

Any element $\Phi \in F$ may be written as

$$
\Phi=(\partial / \partial \theta) \cdot F+\Theta \text {, where } F \in F \text { and } \Theta \in F_{0} .
$$

In the special case where $S O(2)$ acts as a group of rotations on $\mathbf{R}^{2}$ we find, in particular, the well-known result that each $C^{\infty}$ function on $\mathbf{R}^{2}$ in exactly one way can be expressed as the sum of a radial function and a function of the form $\partial F / \partial \theta$ where $F \in C^{\infty}\left(\mathrm{R}^{2}\right)$.

Example 9 (The Lomont-Moses [14] and Cannon-Jordan [3] Cases). The Lie algebra $g=s o(3)$ of the compact semisimple group $G=S O(3)$ is, via standard identifications, spanned by $X_{1}, X_{2}$ and $X_{3} \in g$ where

$$
X_{1}=\left(\begin{array}{ccc}
0 & 0 & 0 \\
0 & 0 & 1 \\
0 & -1 & 0
\end{array}\right), X_{2}=\left(\begin{array}{ccc}
0 & 0 & -1 \\
0 & 0 & 0 \\
1 & 0 & 0
\end{array}\right), X_{3}=\left(\begin{array}{ccc}
0 & 1 & 0 \\
-1 & 0 & 0 \\
0 & 0 & 0
\end{array}\right)
$$

With respect to the scalar product

$$
(X, Y)=-\operatorname{trace}(X Y) \text { for } X, Y \in g,
$$

they form an orthogonal basis of $g$ with $\left\|X_{1}\right\|=\left\|X_{2}\right\|=\left\|X_{3}\right\|=1 / \sqrt{2}$.

Let $\left\{\omega^{1}, \omega^{2}, \omega^{3}\right\}$ be the left-invariant 1 -forms on $G$, dual to $\left\{X_{1} / \sqrt{2}\right.$, $\left.1 / \sqrt{2} X_{2}, 1 / \sqrt{2} X_{3}\right\}$. 
Straightforward computations and the theorem above now imply:

To any linear map $\Phi: s o(3) \rightarrow F$ there are elements $F, F_{1}, F_{2}, F_{3} \in F$ such that

$$
\Phi=d F+\delta\left(\left(\omega^{2} \wedge \omega^{3}\right) \otimes F_{1}+\left(\omega^{3} \wedge \omega^{1}\right) \otimes F_{2}+\left(\omega^{1} \wedge \omega^{2}\right) \otimes F_{3}\right),
$$

or equivalently,

$$
\begin{aligned}
& \Phi\left(X_{1}\right)=X_{1} \cdot F+F_{1}-X_{3} \cdot F_{2}+X_{2} \cdot F_{3}, \\
& \Phi\left(X_{2}\right)=X_{2} \cdot F+F_{2}-X_{1} \cdot F_{3}+X_{3} \cdot F_{1}, \\
& \Phi\left(X_{3}\right)=X_{3} \cdot F+F_{3}-X_{2} \cdot F_{1}+X_{1} \cdot F_{2} .
\end{aligned}
$$

In the Lomont-Moses case $F=C^{\infty}\left(R^{3}\right)$, we can identify the smooth vector fields on $\mathbf{R}^{\mathbf{3}}$ with the maps $\Phi: \mathbf{R}^{\mathbf{3}} \cong g \rightarrow F$, viewing $\Phi$ as the vector field with components $\Phi\left(X_{1}\right), \Phi\left(X_{2}\right)$ and $\Phi\left(X_{3}\right)$. The Cannon-Jordan case can be obtained quite similarly: We let $S O(3)$ act on $C^{\infty}\left(\mathbf{R}^{3} \times \cdots \times \mathbf{R}^{3}\right)$ by diagonal action.

What remains of this section is devoted to proving Theorem 8.

For any fixed $u \in F^{\prime}$ we study the differential 1 -form $\Phi^{u}$ on $G$, defined by

$$
\left\langle\Phi^{u}, X_{g}\right\rangle:=\langle u, g \cdot \Phi(X)\rangle \quad \text { for } g \in G, X \in g .
$$

LEMMA 11. The map $u \mapsto \Phi^{u}$ of $F^{\prime}$ into $\Omega^{2}(G)$ is linear and continuous when $F^{\prime}$ is equipped with the topology of uniform convergence on compact subsets of $F$.

Proof. Trivial from the definition of the topologies and the fact that $G$ acts weakly $C^{\infty}$ on $F$.

By Hodge's theorem $\Phi^{u}$ can in exactly one way be expressed as

$$
\Phi^{u}=d F(u)+\delta \psi(u)+\vartheta(u)
$$

where $F(u) \in C^{\infty}(G), \psi(u) \in \Omega^{2}(G)$ and $\vartheta(u)$ is a harmonic 1 -form on $G$. By Proposition 4 we can even determine $F(u)$ and $\psi(u)$ in such a way that the maps $\Phi^{u} \mapsto F(u), \Phi^{u} \mapsto \psi(u)$ are continuous and linear. We have now proved

LEMma 12. The maps $u \mapsto F(u), u \mapsto \psi(u), u \mapsto \vartheta(u)$ of $F^{\prime}$ into $C^{\infty}(G)$, $\Omega^{2}(G)$ and $\Omega^{1}(G)$ are all continuous and linear when $F^{\prime}$ is equipped with the topology of uniform convergence on compact subsets of $F$.

LEMMA 13. For any $g \in G$ we have

$$
L\left(g^{-1}\right)^{*} F(u)=F(g \cdot u), \quad L\left(g^{-1}\right)^{*} \psi(u)=\psi(g \cdot u), \text { and } L\left(g^{-1}\right)^{*} \vartheta(u)=\vartheta(g \cdot u) \text {. }
$$

Proof. This follows easily from the fact that $L\left(g^{-1}\right)^{*} \Phi^{u}=\Phi^{g \cdot u}$, which was proved in Lemmas 3 and 8. 
LEMMA 14. There are elements $F \in F, \Psi \in \Lambda^{2}(g, F)$ and $\Theta \in\{\Omega \in$ $\Lambda^{1}(g, F) \mid \Omega$ harmonic\} such that for all $g \in G, X, Y \in g$ we have

$$
\begin{gathered}
F(u)(g)=\langle u, g \cdot F\rangle, \\
\psi(u)\left(X_{g}, Y_{g}\right)=\langle u, g \cdot \psi(X, Y)\rangle \quad \text { and } \quad \vartheta(u)\left(X_{g}\right)=\langle u, g \cdot \Theta(X)\rangle .
\end{gathered}
$$

PROOF. Since the three statements are proved in the same way we content ourselves by treating the first one.

As already noted the map $u \mapsto F(u)(g)$ is for any fixed $g \in G$ a continuous linear functional on $F^{\prime}$. Now $F$ is assumed to be quasi-complete and thus [11, $\S 23.9(2)$ ] polar semireflexive so that the dual space of $F^{\prime}$ is $F$. Hence, we see that to each $g \in G$ there exists exactly one element $f(g) \in F$ such that

$$
F(u)(g)=\langle u, f(g)\rangle \quad \text { for all } u \in F^{\prime} .
$$

By Lemma 13 we next see that

or

$$
\begin{aligned}
\left\langle u, f\left(g^{-1} h\right)\right\rangle & =F(u)\left(g^{-1} h\right)=\left(L(g)^{*} F(u)\right)(h) \\
& =F(g \cdot u)(h)=\langle g \cdot u, f(h)\rangle=\left\langle u, g^{-1} f(h)\right\rangle,
\end{aligned}
$$

$$
f(g h)=g \cdot f(h) \text { for all } g, h \in G .
$$

Equivalently $f(g)=g \cdot f(e)$, so that for the $F$ of the lemma, we may take $F=f(e)$.

By the formulae $d\left(\Phi^{u}\right)=(d \Phi)^{u}$, etc., which we established earlier, it follows that

$$
\Phi=d F+\delta \Psi+\Theta .
$$

The uniqueness of the decomposition is also a trivial consequence of the formulae $d\left(\Phi^{u}\right)=(d \Phi)^{u}$ and the uniqueness of the decomposition in Hodge's theorem.

Finally the statement about the semisimple case is a corollary of Lemma 7.

This proves the theorem.

\section{REFERENCES}

1. N. Bourbaki, Éléments de mathématique. Fasc. XXV. Part 1. Livre VI: Intégration. Chap. 6: Intégration vectorielle, Actualités Sci. Indust., no. 1281, Hermann, Paris, 1959. MR 23 \#A2033.

2. H. Cartan and S. Eilenberg, Homological algebra, Princeton Univ. Press, Princeton, N. J., 1956. MR 17, 1040.

3. J. T. Cannon and T. F. Jordan, A no-interaction theorem in classical relativistic Hamiltonian particle dynamics, J. Mathematical Phys. 5 (1964), 299-307. MR 30 \#887.

4. C. Chevalley and S. Eilenberg, Cohomology theory of Lie groups and Lie algebras, Trans. Amer. Math. Soc. 63 (1948), 85-124. MR 9, 567.

5. J. Duistermaat and L. Hörmander, Fourier integral operators. II, Acta Math. 128 (1972), 183-269.

6. W. T. van Est, Group cohomology and Lie algebra cohomology in Lie groups. II, Nederl. Akad. Wetensch. Proc. Ser. A 56 = Indag. Math. 15 (1953), 493-504. MR 15, 505. 
7. S. Helgason, Differential geometry and symmetric spaces, Pure and Appl. Math., vol. 12, Academic Press, New York and London, 1962. MR 26 \#2986.

8. G. Hochschild and G. D. Mostow, Cohomology of Lie groups, Illinois J. Math. 6 (1962), 367-401. MR 26 \#5092.

9. N. Jacobson, Lie algebras, Interscience Tracts in Pure and Appl. Math., no. 10, Interscience, New York and London, 1962. MR 26 \#1345.

10. J. B. Keller, Simple proofs of the theorems of J. S. Lomont and H. E. Moses on the decomposition and representations of vector fields, Comm. Pure Appl. Math. 14 (1961), 77-80. MR 23 \#B3.

11. G. Köthe, Topologische linear Räume. I, Die Grundlehren der math. Wissenschaften, Band 107, Springer-Verlag, Berlin, 1960; 2nd ed., 1966; English transl., Die Grundlehren der math. Wissenschaften, Band 159, Springer-Verlag, New York, 1969. MR 24 \#A411; 33 \#3069; 40 \#1750.

12. H. Leutwyler, Group-theoretical basis of the angular momentum Helmholtz theorem of Lomont and Moses, Nuovo Cimento 37 (1965), 543-555.

13. - A no-interaction theorem in classical relativistic Hamiltonian particle mechanics, Nuovo Cimento 37 (1965), 556-567.

14. J. S. Lomont and H. E. Moses, An angular momentum Helmholtz theorem, Comm. Pure Appl. Math. 14 (1961), 69-76. MR 23 \#B2.

15. O. Loos, Symmetric spaces. I: General theory, Benjamin, New York and Amersterdam, 1969. MR 39 \#365a.

16. M. S. Ragunathan, Discrete subgroups of Lie groups, Ergebnisse der Mathematik und ihrer Grenzgebiete, Band 68, Springer-Verlag, New York, 1972.

17. V. S. Varadajaran, Geomerty of quantum theory. II, Van Nostrand, Princeton, N. J., 1970.

DEPARTMENT OF MATHEMATICS, UNIVERSITY OF AARHUS, AARHUS, DENMARK 